\title{
Extended BEG model of halogenated methanes physisorbed on ionic crystals
}

\author{
T.E. Burns, ${ }^{\mathrm{a}, *}$ J.R. Dennison, ${ }^{\mathrm{b}}$ and Jason Kite ${ }^{\mathrm{b}}$ \\ ${ }^{a}$ Coastal Carolina University, Dept of Chemistry and Physics, Conway, SC 29579 \\ ${ }^{\mathrm{b}}$ Department of Physics, Utah State University, Logan, UT 84322-4415
}

(Received

\begin{abstract}
The 2D dielectric phases and phase transitions of adsorbed dipolar molecules are modeled using a dilute spin-one Ising model. This model is studied in the Blume-Emery-Griffiths formalism, using a mean-field approximation, where the interaction parameters are related to system interaction energies using a unique averaging procedure. The model is applied to four halogenated methane species physisorbed on $\mathrm{MgO}(100)$ and $\mathrm{NaCl}(100)$ surfaces using previous experimental and theoretical studies to estimate the interaction energy parameters. We find that temperature- and coverage-dependent antiferroelectric to ferroelectric, coverage-dependant ferroelectric up to ferroelectric down, reentrant ferroelectric to ferroelectric, and order-disorder dipole phase transitions can occur. Phase diagrams based on this model are presented.
\end{abstract}

PACS Number: 05.50.+q, 64.60.Cn, 64.60.-i, 68.35.Rh, 75.70.Ak, 77.55.+f, 77.80.-e

Keywords: Surface thermodynamics; Ising models; Dipolar molecules; methyl halides; halogenated methanes; Magnesium oxides; Sodium chloride; Physical Adsorption.

*Corresponding author: (843) 349-2225. FAX (843) 349-2841. E-mail: tburns@coastal.edu. 


\section{INTRODUCTION}

Monolayer adsorption of asymmetric molecules can lead to interesting observable phases and phase transitions that depend on adsorbate orientation. Such orientation-dependent interactions can result from steric, magnetic, electric, or chemical asymmetries of the adsorbates. Both the binding energy to the substrate and the interaction energy between admolecules can depend on adsorbate orientation. Dipolar adsorbates, such as $\mathrm{CO}$ and the halogenated methanes, are a particularly interesting class of asymmetric adsorbates which have received considerable attention in recent years.[1, 2] One can study these systems from a single-molecule, quantum mechanical perspective to understand how interactions drive the orientation; however, to extend such studies to the entire adlayer quickly becomes cumbersome and computationally expensive. In this paper, we take the opposite approach: we carefully define the intramolecular and molecule-substrate potentials and use them to apply a simple spin-1 Ising model for adsorption of dipolar molecules with two allowed dipole orientations in the mean field approximation. In this way, we can look for collective behavior that is due to these interactions, and get information about the possible orientationally-ordered phases for these systems. Additionally, the parameter space and hence phase diagrams defined by these potentials have not been previously studied in the Blume-EmeryGriffiths (BEG) approximation; the rich phase behavior we demonstrate below is therefore of more general interest.

Earlier theoretical work focussed on developing a simple model that predicts, for the adsorption of dipoles on a square lattice with only two allowed orientations, which 2D dielectric phases will exist and the approximate range of transition temperatures.[3] This spin lattice model was applied to two specific, well-studied dipolar physisorption systems: $\mathrm{CO}$ on $\mathrm{MgO}(100)$ and $\mathrm{CO}$ on $\mathrm{NaCl}(100)$. For these systems, we found that adsorbate-substrate interactions dominated the system, and determined ordered ferroelectric phases for most temperature and coverage combinations. In both cases, asymmetry in the binding energy determined that the adsorption of the CO molecules with the C-down was energetically preferred, and stable over most experimentally accessible temperatures. For both adsorbate orientations, the CO molecule polarizes such that the net dipole moment is aligned with the substrate electric field. For 
the C-down orientation, the induced dipole $\left(\mathrm{p}_{\text {ind }}\right)$ enhances the natural dipole moment $\left(\mathrm{p}_{\text {nat }}\right)$, causing this to be the energetically favored orientation. For the O-down orientation, the induced dipole is larger than the natural dipole moment. Therefore, in either case, the net dipole moment aligns with the substrate field and antiferroelectric phases are not possible.[3]

An antiferroelectric (AFE) state in a physisorbed system requires $\mathrm{p}_{\text {ind }}<\mathrm{p}_{\text {nat }}$ so that the two vertical orientations have opposed net dipoles, and alternating alignment is energetically favored. Two ways to realize such a system would be to adsorb molecules with a smaller polarizability, and hence a smaller induced dipole moment, or to adsorb molecules with a larger natural dipole moment. For these reasons, we chose $\mathrm{CX}_{\mathrm{n}} \mathrm{Y}_{\mathrm{n}-4}$ dipolar molecules for study.[4] Of particular interest are halogenated methanes (molecules of the form $\mathrm{CX}_{n} \mathrm{Y}_{4-\mathrm{n}}$ with $\mathrm{n}=1,2,3$ and, $\mathrm{Y}=\{\mathrm{H}, \mathrm{F}, \mathrm{Cl}, \mathrm{Br}$, or I $\}$ ) which exhibit large natural dipole moments compared to CO (see Table 1).[5-27] The adsorption of these molecules on graphite has been studied extensively (see reference 24 for a comprehensive review). Antiferroelectrically ordered phases on graphite have been observed for $\mathrm{CF}_{3} \mathrm{H},[14]$ and for $\mathrm{CH}_{3} \mathrm{Cl}$ and $\mathrm{CH}_{3} \mathrm{Br}$.[5] Other electrically ordered phases have been observed for $\mathrm{CF}_{3} \mathrm{Cl}$ on graphite.[28] For graphite coated with $\mathrm{Xe}, \mathrm{CH}_{3} \mathrm{~F}$ is observed to have a ferroelectric phase not seen on bare graphite.[14] However, none of these systems have cubic symmetry and do not lend themselves easily to application of our simple model.

The goal of this work is to apply our model to the halogenated methanes adsorbed on ionic crystals with square lattices. We determine the energy parameters for the Ising model Hamiltonian from interaction energy calculations based on previous experimental and theoretical studies of the two systems. Predicted phase diagrams for these systems based on our simple model are presented.

\section{SPIN-LATTICE MODEL}

The adsorption of dipolar molecules with only two allowed dipole orientations can be modeled as a 2D spin-lattice problem using a spin-1 Ising model.[3] For the dipole adsorption systems studied, we assign a spin $S_{i}=1\left[S_{i}=-1\right.$ ] to a molecule adsorbed with the natural dipole up [down] at a lattice site $\mathrm{i}$ and a spin $S_{i}=0$ to an empty lattice site i [see Fig 1(a)]. The spin-1 system, when limited to ferroelectric 
solutions, has two kinematically coupled order parameters: $<\mathrm{S}>$ for the spin-lattice system and $<\mathrm{S}^{2}>$ for the lattice-gas system. Antiferroelectric ordering can be considered by splitting the lattice into two interpenetrating sublattices, labeled $\mathbf{u}$ and d.[29,30] The antiferroelectric model has four order parameters: the average spins $<\mathrm{S}>$ on the $\mathbf{u}$ and $\mathbf{d}$ sublattices, and the average site occupations $<\mathrm{S}^{2}>$ on the $\mathbf{u}$ and $\mathbf{d}$ sublattices. Complete antiferroelectric ordering occurs when all spins on one sublattice are aligned and all spins on the other sublattice are antialigned, e.g. when $<\mathrm{S}_{\mathrm{u}}>=+1$ and $<\mathrm{S}_{\mathrm{d}}>=-1$.

$$
\begin{aligned}
& \text { Our } \\
& \text { model } \\
& \text { Hamiltonian } \\
& \text { for } \\
& \text { the } \\
& \text { full } \\
& H \equiv H_{\text {int }}+H_{\text {sub }} \\
& =-\sum_{<i, j>}\left[J S_{u_{i}} S_{d_{j}}+K S_{u_{i}}{ }^{2} S_{d_{j}}{ }^{2}+L\left(S_{u_{i}} S_{d_{j}}{ }^{2}+S_{u_{i}}{ }^{2} S_{d_{j}}\right)\right]-\sum_{i}\left[\Delta\left(S_{u_{i}}{ }^{2}+S_{d_{i}}{ }^{2}\right)+H\left(S_{u_{i}}+S_{d_{i}}\right)\right]
\end{aligned}
$$$$
\text { lattice, }
$$

is an extended BEG model.[31] This is the most general Hamiltonian possible for a spin-1 Ising system.[32] This Hamiltonian $\mathrm{H}$ is the sum of a summation over nearest neighbors $<\mathrm{i}, \mathrm{j}>$ of the interaction energy between the admolecules $\left(\mathrm{H}_{\text {int }}\right)$, and a summation over all lattice sites $\mathrm{i}$ of the adsorbate-substrate energies $\left(\mathrm{H}_{\text {sub }}\right)$. Both summations depend on the specific details of the spin orientations where

$$
\begin{aligned}
& \mathrm{J}=1 / 4\left(\mathrm{~J}_{\mathrm{uu}}+\mathrm{J}_{\mathrm{dd}}-2 \mathrm{~J}_{\mathrm{ud}}\right), \\
& \mathrm{K}=1 / 4\left(\mathrm{~J}_{\mathrm{uu}}+\mathrm{J}_{\mathrm{dd}}+2 \mathrm{~J}_{\mathrm{ud}}\right), \\
& \mathrm{L}=1 / 4\left(\mathrm{~J}_{\mathrm{uu}}-\mathrm{J}_{\mathrm{dd}}\right), \\
& \Delta=1 / 2\left(\mu_{\mathrm{u}}+\mu_{\mathrm{d}}\right), \\
& \mathrm{H}=1 / 2\left(\mu_{\mathrm{u}}-\mu_{\mathrm{d}}\right)=(1 / 2 \beta) \ln \left\{\left[1+\exp \left(-\varepsilon_{\mathrm{bd}} \beta\right)\right] /\left[1+\exp \left(-\varepsilon_{\mathrm{bu}} \beta\right)\right]\right\}, \text { with } \\
& \mu_{u(d)}=\frac{1}{\beta} \ln \left(\frac{\theta}{1+\theta}\right)
\end{aligned}
$$

and where $\beta \equiv\left(k_{B} T\right)^{-1}$, $J_{u u}\left(J_{d d}\right)$ is the interaction energy between two neighboring molecules adsorbed spinup (spin-down) [see Figs 1(b) (i) and (iii)], $\mathrm{J}_{\mathrm{ud}}$ is the interaction energy between neighboring molecules adsorbed with spins in opposition [one spin-up and one spin-down, see Fig 1(b) (ii)], $\mu_{\mathrm{u}}\left(\mu_{\mathrm{d}}\right)$ is the 
chemical potential of a molecule adsorbed spin-up (spin-down) [Fig 1(a)], and $\varepsilon_{\mathrm{bu}}>0$ ( $\varepsilon_{\mathrm{bd}}>0$ ) is the binding energy of a molecule adsorbed spin-up (spin-down).

The expectation value of the energy can be found by minimizing the Hamiltonian in Eq. (1) using a density matrix formalism. General analytic expressions, in the mean field approximation, for the average dipole orientations (spin), the coverages, and their ratios are: [32]

$$
\begin{gathered}
M\left(\begin{array}{l}
u \\
d
\end{array}\right) \equiv<S\left(\begin{array}{l}
u \\
d
\end{array}\right)>=\frac{2 \exp \left[\beta\left(\operatorname{LzM}\left(\begin{array}{l}
d \\
u
\end{array}\right)+K z \theta\left(\begin{array}{l}
d \\
u
\end{array}\right)+\Delta\right)\right] \sinh \left[\beta\left(\operatorname{JzM}\left(\begin{array}{l}
d \\
u
\end{array}\right)+\operatorname{Lz} \theta\left(\begin{array}{l}
d \\
u
\end{array}\right)+H\right)\right]}{1+2 \exp \left[\beta\left(\operatorname{LzM}\left(\begin{array}{l}
d \\
u
\end{array}\right)+K z \theta\left(\begin{array}{l}
d \\
u
\end{array}\right)+\Delta\right)\right] \cosh \left[\beta\left(\operatorname{JzM}\left(\begin{array}{l}
d \\
u
\end{array}\right)+\operatorname{Lz} \theta\left(\begin{array}{l}
d \\
u
\end{array}\right)+H\right)\right]} \\
\theta\left(\begin{array}{l}
u \\
d
\end{array}\right)=<S_{\left(\begin{array}{l}
u \\
d
\end{array}\right)}^{2}>=\frac{2 \exp \left[\beta\left(\operatorname{LzM}\left(\begin{array}{l}
d \\
u
\end{array}\right)+K z \theta\left(\begin{array}{l}
d \\
u
\end{array}\right)+\Delta\right)\right] \cosh \left[\beta\left(J z M\left(\begin{array}{l}
d \\
u
\end{array}\right)+\operatorname{Lz} \theta\left(\begin{array}{l}
d \\
u
\end{array}\right)+H\right)\right]}{1+2 \exp \left[\beta\left(\operatorname{LzM}\left(\begin{array}{l}
d \\
u
\end{array}\right)+K z \theta\left(\begin{array}{l}
d \\
u
\end{array}\right)+\Delta\right)\right] \cosh \left[\beta\left(J z M\left(\begin{array}{l}
d \\
u
\end{array}\right)+L z \theta\left(\begin{array}{l}
d \\
u
\end{array}\right)+H\right)\right]}
\end{gathered}
$$

$$
\frac{M\left(\begin{array}{l}
u \\
d
\end{array}\right)}{\theta\left(\begin{array}{l}
u \\
d
\end{array}\right)}=\tanh \left[\beta\left(J z M\left(\begin{array}{l}
d \\
u
\end{array}\right)+L z \theta\left(\begin{array}{l}
d \\
u
\end{array}\right)+H\right)\right]
$$

$\mathrm{M}$ and $\theta$ are found by solving these transcendental equations. The thermodynamics follow from the expressions [32] for the equilibrium free energy

$\phi_{\mathrm{E}}=\beta^{-1} \ln \left[\left(1-\theta_{\mathrm{u}}\right)\left(1-\theta_{\mathrm{d}}\right)\right]+\mathrm{z}\left[\mathrm{JM}_{\mathrm{u}} \mathrm{M}_{\mathrm{d}}+\mathrm{L}\left(\mathrm{M}_{\mathrm{u}} \theta_{\mathrm{d}}+\mathrm{M}_{\mathrm{d}} \theta_{\mathrm{u}}\right)+\mathrm{K} \theta_{\mathrm{u}} \theta_{\mathrm{d}}\right]$

and the pressure $\mathrm{p}=-\phi_{\mathrm{E}}$. A more complete description of this spin lattice model can be found in references 3 and 33.

\section{APPLICATION TO HALOGENATED METHANES ON NaCl (100) AND ON MgO (100)}

We now apply the model specifically to halogenated methanes on $\mathrm{MgO}(100)$ and $\mathrm{NaCl}(100)$ surfaces. To apply our spin-lattice model, the interaction energy parameters J, K and L must be evaluated in the mean field approximation and under the appropriate conditions. Details of the application to the general case are discussed elsewhere.[3] 
For our present application, the binding energies for both dipole orientations favor adsorption, and we choose the zero of energy such that these are negative. From Eqns. 2, we see that this implies $\mu_{\mathrm{u}}$, $\mu_{\mathrm{d}}<0$, and that $\Delta$ is always negative. $\mathrm{H}$ is a direct measure of the binding energy difference between the two possible dipole orientations. We choose our definition of "up" spin such that $\mathrm{H}$ is positive for all the systems considered here. Antiferroelectric or ferrielectric states require $\mathrm{J}<0$ (that is, $2 \mathrm{~J}_{\mathrm{ud}}>\mathrm{J}_{\mathrm{uu}}+\mathrm{J}_{\mathrm{dd}}$ ). This implies that the interaction energy between two opposed dipoles [see Fig. 1(b) (ii)] is less than the interaction energy of at least one of the orientations with two aligned dipoles [Figs. 1(b) (i) and (iii)]. K is a measure of the total energy in the interactions, while $\mathrm{L}$ is measure of the difference in energy for the two parallel orientations (up-up, and down-down). Note that $\Delta$ and $\mathrm{K}$ do not play a role in the transcendental equation (5) we solve for the average spin and occupation, and are simply additive in Eqns. (3) and (4). $\Delta$ and $\mathrm{K}$ act to set the energy scales for the chemical potential and interaction energies, respectively, and do not determine the allowed phases. This is determined through $\mathrm{J}, \mathrm{L}$, and $\mathrm{H}$. Note that $\mathrm{H}$ is dependant on $\mathrm{T}$, but not on $\theta$, while $\mathrm{J}$ and $\mathrm{L}$ depend on $\theta$ but not $T$. If $|J|>\mathrm{H}$ or $\mathrm{L}$, so $\mathrm{J}$ is the dominant term, AFE phases are found. If $\mathrm{H}$ or $\mathrm{L}$ is dominant, a FE phase is preferred, and the orientation is driven by the energy differences, i.e., a positive (negative) $\mathrm{H}$ or L favors FE-up (-down) orientation. To apply our model and determine the possible 2D dielectric phases and phase transitions for the two systems, we calculate the microscopic potential, and evaluate the energy parameters in Eq. (2).

\section{A. Determination of Substrate Field Strength Parameters}

Both $\mathrm{NaCl}$ and $\mathrm{MgO}$ have a rock salt structure, with lattice constants a $=0.564 \mathrm{~nm}$, and $\mathrm{a}=$ $0.421 \mathrm{~nm}$, respectively. The adsorbate-substrate field strength parameters $\Delta$ and $\mathrm{H}$ are determined directly from binding energy calculations (see Table 1). As expected, the binding energy is dependent on adsorbate orientation and on adsorbate position due to substrate potential corrugation. To fulfill the model requirement of only two allowed energy states, we limit our application to vertical adsorption above cation sites with either up or down alignment [see Fig 1(a)]. This restricts our study to adsorbed phases with commensurate, square-symmetric lattices. 
The binding energies for $\mathrm{CH}_{3} \mathrm{~F}$ on $\mathrm{NaCl}$, as well as $\mathrm{CH}_{3} \mathrm{Cl}$, and $\mathrm{CH}_{3} \mathrm{I}$ on $\mathrm{MgO}$ have been measured experimentally.[34, 35] We estimate the binding energy for $\mathrm{CH}_{3} \mathrm{Br}$ on $\mathrm{MgO}$ by linearly interpolating the trend in binding energies for the measured halogenated methanes (Table 2). The additional binding energies are estimated by assuming that the ratio of the binding energy of a halogenated methane on $\mathrm{MgO}$ to that on $\mathrm{NaCl}$ scales as the ratio of the binding energies of $\mathrm{CO}$ on $\mathrm{MgO}$ to that on $\mathrm{NaCl}$, known from earlier work (see Ref. 22 and the references within). Binding energy asymmetry is estimated to arise from asymmetric dipole-substrate interactions (Table 2).[34-37] Depending on dipole orientation, the induced dipole will reinforce or mitigate the binding energy by an amount that is proportional to the square of the induced dipole moment and inversely proportional to the molecular polarizability $\alpha$, i.e, $\varepsilon_{\text {up } / \text { down }}=\varepsilon_{\mathrm{b}} \pm\left[\mathrm{p}_{\text {ind }}{ }^{2} / 2 \cdot \alpha\right]$; this contribution is calculated and tallied in Table 2. The adsorbate-substrate field strength parameters $\Delta$ and $\mathrm{H}$ are then calculated directly from Eq. 2.

\section{B. Calculation of Adsorbate Interaction Energy Parameters}

The natural dipole moments for all halogenated methanes are experimentally known. The induced dipole moment arises from the interaction of the molecule with the substrate electric field. By assuming that to first order the substrate electric field is not perturbed by the adsorbed molecule, we can estimate the induced dipole for the halogenated methanes on $\mathrm{NaCl}$ and $\mathrm{MgO}$ by scaling the induced dipole for $\mathrm{CO}$ on $\mathrm{NaCl}$ and $\mathrm{MgO}$ by the ratio of the polarizabilities, i.e., $\mathrm{p}_{\text {ind }}=\alpha \cdot\left(\mathrm{p}_{\mathrm{CO}} / \alpha_{\mathrm{CO}}\right)$.. This induced dipole will either enhance or mitigate the natural dipole moment, i.e., $\mathrm{p}_{\mathrm{u} / \mathrm{d}}=\mathrm{p}_{\text {ind }} \pm \mathrm{p}_{\text {nat }}$. Here we find that for $\mathrm{CH}_{3} \mathrm{~F}, \mathrm{CH}_{3} \mathrm{Cl}$, and $\mathrm{CH}_{3} \mathrm{Br}$ on both substrates, and $\mathrm{CH}_{3} \mathrm{I}$ on $\mathrm{NaCl}$, $\mathrm{p}_{\text {ind }}<\mathrm{p}_{\text {nat }}$ so that the dipole moment for the up-orientation points away from the substrate surface, while the dipole moment for the down-orientation points towards the surface, allowing for the possibility of AFE phases. For $\mathrm{CH}_{3} \mathrm{I}$ on MgO, $\mathrm{p}_{\text {ind }}>\mathrm{p}_{\text {nat, }}$ and both dipole orientations point away from the substrate surface; AFE ordering can occur, however, if the interaction energy for the two opposed dipoles is favored over two aligned dipoles, leading to a negative $\mathrm{J}$. 


\section{Determination of Hamiltonian Interaction Parameters}

We now relate the physical parameters such as dipole moment to the interaction parameters $\mathrm{J}$, K, and $\mathrm{L}$, found in the system Hamiltonian. The average interaction energy, $<\mathscr{F} / \mathrm{int}>$, is related to $\mathrm{J}_{\mathrm{uu}}, \mathrm{J}_{\mathrm{dd}}, \mathrm{J}_{\mathrm{ud}}, \mu_{\mathrm{u}}$ and $\mu_{\mathrm{d}}$ through the following equation:[3]

$$
\begin{aligned}
& U_{\text {int }}=\frac{\left\langle H_{\text {int }}\left(M_{d}, M_{u}, \theta_{d}, \theta_{u}\right)\right\rangle}{N} \\
& =-Z\left\{J M_{u} M_{d}+K \theta_{u} \theta_{d}+L\left(M_{u} \theta_{d}+\theta_{u} M_{d}\right)\right\}
\end{aligned}
$$

$\mathrm{U}_{\text {int }}$ can be calculated for specific sublattice configurations and as a function of coverage. For example, we can assume that both sublattices are oriented spin up $(\mathrm{M}=+\theta)$, both sublattices are oriented spin down $(\mathrm{M}=-\theta)$, or one sublattice is entirely spin-up, and one entirely spin-down (antiferroelectric configuration, $\mathrm{M}=0$ ). By applying the relationships found in Eq. 2, we find:[3]

$$
\begin{array}{lll}
U_{\mathrm{int}}^{+}=\frac{\left\langle H_{\mathrm{int}}^{+}(M, \theta)\right\rangle}{N}=\frac{-\mathrm{z} \theta^{2}}{2}(J+2 L+K) & =\frac{-\mathrm{z} \theta^{2}}{2} J_{\text {uи }} & ; M=+\theta \\
U_{\mathrm{int}}^{0}=\frac{\left\langle H_{\mathrm{int}}^{0}(M, \theta)\right\rangle}{N}=\frac{-\mathrm{z} \theta^{2}}{2}(K) & =\frac{-\mathrm{z} \theta^{2}}{8}\left(J_{\text {uu }}+J_{d d}+2 J_{u d}\right) & ; M=0 \\
U_{\mathrm{int}}^{-}=\frac{\left\langle H_{\mathrm{int}}^{-}(M, \theta)\right\rangle}{N}=\frac{-\mathrm{z} \theta^{2}}{2}(J-2 L+K) & =-\frac{z \theta^{2}}{2} J_{d d} & ; M=-\theta
\end{array}
$$

We calculate $\mathrm{U}_{\text {int }}(=<\mathscr{O}$ int $>/ \mathrm{N})$ for the three special lattices, using the physical parameters discussed above:

$$
\begin{gathered}
U_{i n t}^{i}=\frac{1}{32 \pi \varepsilon_{o}} \sum_{j \neq i}\left[\frac{\left(p_{u}-p_{d}\right)^{2}}{r_{i j}^{3}} S_{i} S_{j}\right] \\
+\frac{1}{8 \pi \varepsilon_{o}} \sum_{j \neq i} \frac{q_{i} q_{j}}{r_{i j}^{5}} S_{i}^{2} S_{j}^{2}+\frac{1}{2} \sum_{j \neq i} 4\left[\varepsilon_{l j}\left(\frac{\sigma}{r}\right)^{12}-\varepsilon_{l j}\left(\frac{\sigma}{r}\right)^{6}-2 D\left(\frac{\sigma}{r}\right)^{12}\right] S_{i}^{2} S_{j}^{2}
\end{gathered}
$$

The first term is the interaction energy of an infinite raft of dipoles, where $\mathrm{p}_{u}$ and $\mathrm{p}_{\mathrm{d}}$ are the dipole moments, $\mathrm{r}_{\mathrm{ij}}$ is the distance from a central dipole, and $\varepsilon_{0}$ is the permittivity of free space. The second term is the interaction energy of an infinite raft of quadrupoles oriented parallel to one another, where $q_{i}$ is the 
quadrupole moment of the atom on the $\mathrm{i}^{\text {th }}$ site; note that the interaction energy of the dipole and quadrupole moments is zero when the molecules are limited to parallel or antiparallel orientation. The third term is the dispersive energy; this takes into account both the Lennard-Jones interactions as given by the parameters, $\varepsilon$ and $\sigma$, defined in Table 1 , and a contribution due to the shape of the molecules. This steric term is quantified by the Buckingham-Pople factor, $\mathrm{D}$, which is positive and between 0 and 0.5 for rod-like molecules like the halogenated methanes.[5] This factor was experimentally determined for $\mathrm{CH}_{3} \mathrm{~F}$ and $\mathrm{CH}_{3} \mathrm{Cl},[15,17]$ and the trend is linearly extrapolated for other halogenated methanes.

We calculate $U_{\text {int }}$ for the 44 most dense commensurate superlattices where $\theta_{\max }=1$ and $\theta_{\min }=0.011$ $(\theta=1$ corresponds to one adsorbate molecule for each cation site) for the three specific sublattice configurations identified in Eq. 8. The summations are exact for the first 100 nearest-neighbor shells, and corrected for the rest of an infinite lattice with an effective medium approximation.[3] We then solve Eqns. 8 for $\mathrm{J}, \mathrm{K}, \mathrm{L}, \mathrm{H}$, and $\Delta$ as a function of $\theta$. These parameters are then used to solve Eqns. 3, 4, and 5 for the magnetization on sublattices $\mathbf{u}$ and $\mathbf{d}$, as a function of temperature for each sublattice.

\section{Phase Changes in Dipole Orientation}

By solving Eq. 3 for $\mathrm{M}$ as a function of temperature at a variety of coverages, we can probe the thermodynamics and phase transitions of these systems. For each system, we generate a family of net normalized spin (magnetization per unit coverage) plots for both sublattices. Two representative sets for $\mathrm{CH}_{3} \mathrm{~F}$ and $\mathrm{CH}_{3} \mathrm{I}$ on $\mathrm{MgO}$ are shown in Figs. 2 and 3, respectively; from these we can determine the phase of the system as a function of temperature for the coverages considered. Ferroelectric (FE) phases exhibit degenerate sublattice magnetization curves. Antiferroelectric (AF) phases have equal magnitude and opposite sign magnetization curves, while ferroelectric (fe) and antiferrielectric (af) phases have dissimilar magnitudes and equal or opposite signs, respectively. Bifurcation of the magnetization curves is indicative of a transition from a ferroelectric phase to a ferrielectric phase. The antiferrielectric and ferrielectric phases both imply two imbedded FE ordered states with different average spins; note that because we look only for average spin, we cannot determine if some local orientationally-ordered pattern or super cell gives rise to this behavior. Also note that at high temperature all systems relax to a spin- 
disordered (D) phase with both sublattice spin curves approaching zero. In order to determine the phase of the system at a particular temperature and coverage, we compare the average normalized spin on each sublattice and subject them to the following specific criteria. If the magnitude of the average spin on each sublattice is the same to within a tolerance of $<1 \%$, the phase is a ferro phase; if the signs of the average spins are the same (different), it is FE (AF). When the magnitudes of the average spin differ by $>1 \%$, the phase is a ferri phase; if the signs are the same (different), the phase is fe (af). These phases are further distinguished as up (u) or down (d) if the total net spin $M_{\mathbf{u}}+M_{\mathbf{d}}$ is positive or negative, respectively. Finally, the spin-disordered (D) phase is defined to be when the average net normalized spins of both sublattices are $<1 \%$. [Note that the $\mathbf{A F}$ and $\mathbf{D}$ phases are not designated as up or down, since the total net normalized spin is $<1 \%$.]

In this way, phase diagrams as a function of temperature and coverage for the four halogenated methanes on $\mathrm{NaCl}$ and $\mathrm{MgO}$ are built and presented in Figs. 4 and 5, respectively. In these figures, the phase is represented by a gray-scale patch at each of 301 temperatures and 44 coverages studied. Note that the unshaded bands in the phase diagrams indicate unstudied regions where there are no commensurate square-symmetric lattices. We find numerous phases and phase transitions are theoretically possible that depend both on temperature and coverage, as enumerated below.

The phase diagrams for all of the halogenated methanes adsorbed on $\mathrm{NaCl}$ are very similar (see Fig. 3). They exhibit a low-temperature, low-coverage AF phase. This transitions with increasing temperature first to an afd phase, followed by a fed phase, and then a FEd phase at the highest temperatures. Almost all phase transition temperatures decrease with decreasing coverage. This is expected because the dipole-dipole interactions are weaker at low coverage, where the molecules are more widely separated; it requires less energy to make the transition. The FEd phase transitions to a disordered phase as the temperature increases further, above temperatures shown in Figs. 4 and 5. Note that the exact transition coverages and temperatures vary from system to system (see Fig. 4 for details).

The halogenated methanes adsorbed on MgO exhibit particularly rich phase diagrams. Each of the four phase diagrams shown in Fig. 5 have seven phases present. At low coverage and temperature, 
the systems exhibits an FEu phase. At higher coverage and temperature, the four systems exhibit a FEd phase, transitioning as temperature decreases to a fed, then afd, and finally $\mathbf{A F}$ phase. [Note, for $\mathrm{CH}_{3} \mathrm{I}$ on MgO at highest coverage, only the FEd phase is present.] At intermediate coverages, the phase diagram is much more complex. For each of the four systems, a pinwheel-like arrangement of phases is predicted, starting with an FEd phase at high coverage and temperature and proceeding to fed, afd, AF, afu, feu, and FEu phases moving counter-clockwise. Finally, a disordered phase exists between the high temperature FE phases. Of particular interest is the reentrant behavior as the phase of each $\mathrm{MgO}$ adsorbate system progresses from FEu to feu and then back to FEu as temperature increases. This reentrant behavior has a distinctive loop signature in the net normalized spin curves of Fig. 2 and 3. Similar interesting behavior is found for the $\mathrm{CH}_{3} \mathrm{I}$ on $\mathrm{MgO}$ system as the phases progress with increasing coverage from FEd to fed to afd and then back to fed and finally FEd.

\section{DISCUSSION AND CONCLUSIONS}

We estimate that changes of $\approx 20 \%$ in $|\mathrm{M}|$ should be experimentally observable. Spectral shifts in IR peaks have been observed and attributed to different orientations for adsorbed species; in particular, a $20-\mathrm{cm}^{-1}(2.5 \mathrm{meV})$ difference in two ir peaks for $\mathrm{CH}_{3} \mathrm{~F}$ adsorbed on $\mathrm{NaCl}$ was associated with either dipole-up or dipole-down adsorption.[38] We could expect similar wave number difference for other methyl halides, and given such large separations, small changes in M would be observable with standard IR spectroscopy (within the limits of intrinsic peak broadening and other effects, such as molecular tilting). The contrast of methyl group and the halide atoms for x-ray, electron or neutron diffraction should be sufficient to readily distinguish the two vertical orientations of the methyl halide.

There is very limited direct information on the structure or phase transitions of the methyl halides on $\mathrm{MgO}$ or $\mathrm{NaCl}$, particularly at lower coverages, to compare with our theoretical model predictions. Xray diffraction studies of $\mathrm{CH}_{3} \mathrm{I}$ on $\mathrm{MgO}$ found a commensurate adlayer for the monolayer, but could not determine either molecular placement or orientation within the unit cell.[39] X-ray diffraction of $\mathrm{CH}_{3} \mathrm{Cl}$ and $\mathrm{CH}_{3} \mathrm{Br}$ also determined a commensurate component to the adlayer, in co-existence with a disordered component. No unit cell or orientational ordering was determined.[39] 
Several methyl halides adsorbed on both $\mathrm{MgO}$ and $\mathrm{NaCl}$ have been studied using temperatureprogrammed desorption (TPD) and time of flight (TOF) mass spectroscopy.[35,40,41] These measurements were used to infer the orientational ordering of the adsorbed layer. $\mathrm{CH}_{3} \mathrm{I}$ was inferred to adsorb perpendicular to the substrate surface on $\mathrm{MgO}$, with a parallel alignment of the adsorbate molecules at low coverage transitioning to islands of adsorbate preferring an anti-parallel alignment as the coverage increases, in agreement with our model. [40,41] They do not observe a transition back to parallel alignment we see in our model.[40,41] TPD and TOF mass spectroscopy measurements of $\mathrm{CH}_{3} \mathrm{I}$ on $\mathrm{NaCl}$ found the molecules tilted with respect to the surface normal. Helium diffraction studies found a rectangular unit cell, with molecules antialigned for $\mathrm{CH}_{3} \mathrm{Br}$ on $\mathrm{NaCl}$ in agreement with our model,[35] while TPD and TOF measured the molecules to be aligned with the dipole moment parallel to the surface.[42]

In general, the agreement between experiment and our model is good, but somewhat limited by the assumptions built into our model. There is experimental evidence that the strong dipole moments do play a significant role in driving the orientational ordering, and we see this in our model as orientational ordering that depends on coverage, temperature, and on the adsorbed species. However, factors such as quadrupolar interactions and the interaction of the dipole moment with the substrate, leading to molecular tilting and rectangular unit cells, clearly play an important role as well. Our model can be refined to account for these in several ways. Detailed calculations of the adsorbate-substrate interactions as a function of both adsorption site and adsorbate orientation would lead to more accurate interaction parameters in our model, and hence more accurate phase diagrams. These calculations could incorporate quantum mechanical effects, making the estimation of the energy parameters even more accurate. Generalizing our model to allow for tilting in the adlayer molecules is straightforward, as all the electrostatic potentials are angle dependent.[33] We could then minimize the interaction energy as a function of angle and coverage, to allow for the tilting inferred from some of the experiments describe above. Generalizing to allow for non-square symmetric unit cells is more challenging, but is also one of our future projects. 


\section{ACKNOWLEDGMENTS}

This work has been supported by the Donors to the Petroleum Research Fund.

\section{REFERENCES}

[1] W. Steele, Chem. Rev. 93 (1993) 2355.

[2] E. Umback; H.-J. Freund, (Springer-Verlag, Berlin, 1993).

[3] T.E. Burns and J.R. Dennison, Surface Science 395 (1998) 46.

[4] T.E. Burns and J.R. Dennison, Bull. Am. Phys. Soc. 40 (1995) 128.

[5] S.A. Pope, Surf. Sci. 139 (1984) 299.

[6] CRC Handbook of Chemistry and Physics, 60th Ed., R.d. Weast, ed., (CRC Press, Boca Raton, FL, 1979), pp. C81-548.

[7] W. Brakeman; A.L. Mossman, Matheson Gas Data Book. (Matheson, Lyndhurst, 1976).

[8] C.D. Burru, J Chem Phys. 28 (1958) 427.

[9] R.D. Amos, Chem Phys Lett 87 (1982) 23.

[10] D.E. Storgyn and A.P. Storgyn, Mol Phys. 11 (1966) 371.

[11] J.E. Gready, G.B. Backsay, and N.S. Hush, Chem. Phys. 31 (1978) 375.

[12] V.I. Minkin, Dipole Moments in Organic Chemistry, (Plenum Press, New York, 1970).

[13] T.B. Lakdar, E. Tailandier, and Berthier G., Mol. Phys. 39 (1980) 881.

[14] P.A. Rowntree, G. Scoles, and J. Ruiz-Suirez, Phys. Chem. 94 (1990) 8511.

[15] T.G. Copeland and R.H. Cole, J Chem Phys. 64 (1976) 1741.

[16] A.S. Casparian and R.H. Cole, J. Chem. Phys. 60 (1974) 1106.

[17] H. Sutter and R.H. Cole, J. Chem. Phys. 52 (1970) 132.

[18] K.E. Ladig, J Chem. Phys. 97 (1993) 12760.

[19] D.A. Dixon, J. Chem Phys. 92 (1988) 86.

[20] E. McCullogh, private communication. Quadrupole moment calculations made using the GAMESS program: Schmidt, M.W., et al., J. Computational Chem., 14 (1993) 1347.

[21] Johri, G. K.; Pathak, S.K., Indian J. Pure and Applied Phys. 29 (1991) 214.

[22] M.P. Bogaard and J. Muenter, J. Mol. Spec. 83 (1980) 279.

[23] T.K. Bose, J.S. Sochanski, and R.H. Cole, J. Chem. Phys. 57 (1972) 3592.

[24] K. Knorr, Phys. Rep. 214 (1992) 115.

[25] M.Y. Kok, A.J. van der Est, and E.E. Burnell, Liquid Crystals 3 (1988) 485.

[26] C.L. Norris, E.F. Pearson, and W.H. Flygare, J Chem. Phys 60, 1758 (1974). 
[27] P.G. del Conde, P.S. Bagus, and C.W. Bauschlicher Jr., Theor Chim Acta 45 (1977) 121.

[28] K. Knorr and W. Wiechert, Phys. Rev. B 37 (1987) 3524.

[29] W. Hoston and A. N. Berker, Phys. Rev. Lett. 67 (1991) 1027.

[30] W. Hoston, Massachusetts Institute of Technology, 1991.

[31] M. Blume, V.J. Emery, and R.B. Griffiths, Phys. Rev. A 4 (1971) 1071.

[32] J. Sivardière and J. Lajzerowicz, Phys. Rev. A 11 (1975) 2090.

[33] T.E. Burns, Utah State University, 1994.

[34] A. Lakhilfi, Mol. Phys. 78 (1993) 659.

[35] G.N. Robinson, J. Chem. Phys. 96 (1992) 9212.

[36] M. Sprung, J.Z. Larese, Phys. Rev. B 61 (2000) 13155.

[37] J.V. Setzler, Z.-H. Huang, and H. Guo, J. Chem. Phys. 103 (1995) 4300.

[38] J. Heidberg, I. Hussla, and Z. Szilagyi, J. of El. Sp. \& Rel. Phen. 30 (1983) 53.

[39] M. Sprung, University of Kiel, 2001.

[40] K.A. Trentlman, D.H. Fairbrother, P.G. Strupp et al., J. Chem. Phys. 96 (9221) 1992.

[41] D. H. Fairbrother, K.A. Briggman, P.C. Stair et al., J. Chem. Phys. 102 (7267) 1995.

[42] S.J. Garrett, D.V. Heyd, and J.C. Polanyi, J. Chem. Phys. 106 (7834) 1997. 


\section{Figure Captions}

Fig. 1. (a) Spin assignment for a dipole adsorbed vertically above a cation lattice site $(+)$ on an ionic crystal surface. (b) Dipole and molecular orientations for the three configurations of $\mathrm{CH}_{3} \mathrm{X}(\mathrm{X}=\mathrm{F}, \mathrm{Cl}$, $\mathrm{Br}$, or I) adsorbed vertically above metal ion lattice sites on ionic crystal surfaces.

Fig. 2. Net normalized spin $(\mathrm{M} / \Theta)$ for both sublattices as a function of temperature for $\mathrm{CH}_{3} \mathrm{~F}$ on $\mathrm{MgO}$. Curves are shown for a series of decreasing coverages: $1.00,0.25,0.077,0.025,0.016,0.015,0.0139$, 0.0137, $0.0135,0.0125,0.0123,0.0122,0.0118,0.0112,0.0099 \mathrm{ML}$. These coverages correspond to the higher temperature curves as spin increases shown in order as purple, dark blue, light blue, green and red and as solid, dashed and dotted lines.

Fig. 3. Net normalized spin $(\mathrm{M} / \Theta)$ for both sublattices as a function of temperature for $\mathrm{CH}_{3} \mathrm{I}$ on $\mathrm{MgO}$. Curves are shown for a series of decreasing coverages: $1.00,0.50,0.25,0.20,0.125,0.050,0.035,0.031$, 0.029, 0.028, 0.027, 0.025, 0.017, 0.0147, $0.0099 \mathrm{ML}$. These coverages correspond to the higher temperature curves as spin increases shown in order as purple, dark blue, light blue, green and red and as solid, dashed and dotted lines.

Fig. 4. Phase diagrams for (a) $\mathrm{CH}_{3} \mathrm{~F}$, (b) $\mathrm{CH}_{3} \mathrm{Cl}$ (c) $\mathrm{CH}_{3} \mathrm{Br}$ and (d) $\mathrm{CH}_{3} \mathrm{I}$ on $\mathrm{NaCl}$ as a function of temperature and coverage. Note that logarithmic scales are used on both axes. Color-coded phases are identified on the $\mathrm{CH}_{3} \mathrm{I}$ phase diagram.

Fig. 5. Phase diagrams for (a) $\mathrm{CH}_{3} \mathrm{~F}$, (b) $\mathrm{CH}_{3} \mathrm{Cl}$ (c) $\mathrm{CH}_{3} \mathrm{Br}$ and (d) $\mathrm{CH}_{3} \mathrm{I}$ on $\mathrm{MgO}$ as a function of temperature and coverage. Note that logarithmic scales are used on both axes. Color-coded phases are identified on the $\mathrm{CH}_{3} \mathrm{I}$ phase diagram. 
Table 1: Adsorbate Parameters

\begin{tabular}{|c|c|c|c|c|c|c|c|c|}
\hline \multirow[t]{3}{*}{ Adsorbate } & \multicolumn{3}{|c|}{ Electrostatic Energy Parameters } & \multicolumn{3}{|c|}{ Dispersive Energy Parameters } & \multicolumn{2}{|c|}{ Bulk Temp. } \\
\hline & \multirow{2}{*}{$\begin{array}{l}\text { Dipole } \\
\text { Moment, } \mathrm{p}_{\text {nat }} \\
\left.\text { (x } 10^{-30} \mathrm{Cm}\right)\end{array}$} & \multirow{2}{*}{$\begin{array}{l}\text { Quadrupole } \\
\text { Moment, q } \\
\left(x 0^{-40} \mathrm{Cm}^{2}\right)\end{array}$} & \multirow{2}{*}{$\begin{array}{l}\text { Polarizability, } \\
\alpha \\
\left(\mathrm{x} 10^{-40} \mathrm{C}^{2} \mathrm{~m}^{2} \mathrm{~J}^{-}\right. \\
\left.{ }^{1}\right)\end{array}$} & \multicolumn{2}{|c|}{ Lennard-Jones Parameters } & \multirow{2}{*}{$\begin{array}{l}\text { Buckingham- } \\
\text { Pople shape } \\
\text { factor, D [a] }\end{array}$} & \multirow{2}{*}{$\begin{array}{l}\mathrm{T}_{3 \mathrm{Dm}} \\
(\mathrm{K}) \\
{[\mathrm{b}]}\end{array}$} & \multirow{2}{*}{$\begin{array}{l}\mathrm{T}_{3 \mathrm{Db}} \\
(\mathrm{K}) \\
{[\mathrm{b}]}\end{array}$} \\
\hline & & & & $\varepsilon_{\mathrm{LJ}}(\mathrm{meV})$ & $\sigma_{\mathrm{LJ}}(\mathrm{nm})$ & & & \\
\hline $\mathrm{CO}$ & $0.374[\mathrm{c}, \mathrm{d}, \mathrm{e}]$ & $8.34[\mathrm{f}]$ & 2.334 & $8.6 \pm 0.9[\mathrm{~g}]$ & $3.68 \pm 0.08[\mathrm{~g}]$ & $+0.40 *$ & 64.1 & 83.1 \\
\hline $\mathrm{CH}_{3} \mathrm{~F}$ & $\begin{array}{l}6.17 \pm 0.07 \\
\text { [d,g,h,i,j,k,l, } \\
\text { m] }\end{array}$ & $\begin{array}{l}1.4 \pm 0.6 \\
{[\mathrm{~d}, \mathrm{~h}, \mathrm{j}, \mathrm{m}, \mathrm{n}, \mathrm{p}]}\end{array}$ & $\begin{array}{l}2.904 \pm 0.07 \\
{[\mathrm{~d}, \mathrm{j}, \mathrm{l}, \mathrm{o}, \mathrm{q}]}\end{array}$ & $\begin{array}{l}17 \pm 1 \\
{[\mathrm{j}, \mathrm{r}]}\end{array}$ & $\begin{array}{l}0.380 \\
\pm 0.005[\mathrm{j}, \mathrm{r}]\end{array}$ & $+0.25[\mathrm{j}]$ & 131.3 & 194.7 \\
\hline $\mathrm{CH}_{3} \mathrm{Cl}$ & $\begin{array}{l}6.31 \pm 0.07 \\
{[\mathrm{~g}, \mathrm{~h}, \mathrm{l}, \mathrm{s}]}\end{array}$ & $7.35[0]$ & $\begin{array}{l}4.7 \pm 0.2 \\
{[1,0, p]}\end{array}$ & $\begin{array}{l}30.4 \pm 0.4 \\
{[1, r]}\end{array}$ & $\begin{array}{l}0.403 \\
\pm 0.008[\mathrm{l}, \mathrm{r}]\end{array}$ & $+0.27[1]$ & 175.4 & 249.2 \\
\hline $\mathrm{CH}_{3} \mathrm{Br}$ & $\begin{array}{l}6.01 \\
{[r, g, i]}\end{array}$ & $\begin{array}{l}15 \pm 4 \\
{[t, u, v]}\end{array}$ & $\begin{array}{l}6.2 \pm 0.5 \\
{[g, p, q, t]}\end{array}$ & $40 *$ & $0.43 *$ & $+0.29 *$ & 199.8 & 276.7 \\
\hline $\mathrm{CH}_{3} \mathrm{I}$ & $\begin{array}{l}5.40 \\
{[h, g]}\end{array}$ & $17.9[\mathrm{t}, \mathrm{v}]$ & $\begin{array}{l}8.92[\mathrm{t}, \mathrm{q}] \text { See } \\
\text { Ref. }\end{array}$ & $50 *$ & $0.55 *$ & $+0.31 *$ & 206.7 & 315.5 \\
\hline
\end{tabular}

* Estimated by extrapolation

[a] See Ref. 4

[b] See Ref. 5

[c] See Ref. 6

[d] See Ref. 7

[e] See Ref. 8

[f] See Ref. 9

[g] See Ref. 10

[h] See Ref. 11

[i] See Ref. 12

[j] See Ref. 13

[k] See Ref. 14

[l] See Ref. 15

[m] See Ref. 16 [n] See Ref. 17

[o] See Ref. 18

[p] See Ref. 10

[q] See Ref. 20

[r] See Ref. 21

[s] See Ref. 22

[t] See Ref. 23

[u] See Ref. 24

[v] See Ref. 25 
Table 2: Adsorbate/Substrate System Parameters

\begin{tabular}{|c|c|c|c|c|c|c|c|c|c|c|c|}
\hline \multicolumn{3}{|c|}{ Adsorbate/Substrate System } & \multicolumn{3}{|c|}{ Electrostatic Energies } & \multirow{2}{*}{\multicolumn{3}{|c|}{$\begin{array}{c}\text { Binding Energy Parameters } \\
\text { Binding energies }\end{array}$}} & \multicolumn{3}{|c|}{ Relevant Temperatures } \\
\hline \multirow{2}{*}{$\begin{array}{l}\text { Adsorbate/ } \\
\text { Substrate }\end{array}$} & \multirow{2}{*}{$\begin{array}{l}\text { Cation } \\
\text { Spacing, } \\
\mathrm{a}_{\mathrm{o}}(\mathrm{nm})\end{array}$} & \multirow{2}{*}{$\begin{array}{l}\text { Max. } \\
\text { Coverage } \\
(\mathrm{ML})\end{array}$} & \multirow{2}{*}{$\begin{array}{l}\text { Induced } \\
\text { Dipole } \\
\text { Moment, } \mathrm{p}_{\text {ind }} \\
\text { (x } 10^{-30} \mathrm{Cm} \text { ) }\end{array}$} & \multirow{2}{*}{$\begin{array}{l}\text { Up dipole } \\
\text { Moment, } \mathrm{p}_{\mathrm{up}} \\
\left.\text { (x } 10^{-30} \mathrm{Cm}\right)\end{array}$} & \multirow{2}{*}{$\begin{array}{l}\text { Down dipole } \\
\text { Moment, } \mathrm{p}_{\text {down }} \\
\left.\text { (x } 10^{-30} \mathrm{Cm}\right)\end{array}$} & & & & & & Bulk \\
\hline & & & & & & $\begin{array}{l}\varepsilon_{\mathrm{b}} \\
(\mathrm{meV})\end{array}$ & $\begin{array}{l}\varepsilon_{\text {up }} \\
\text { (meV) }\end{array}$ & $\begin{array}{l}\varepsilon_{\text {down }} \\
(\mathrm{meV})\end{array}$ & $\begin{array}{l}\text { Meltin } \\
\mathrm{g}, \mathrm{T}_{2 \mathrm{Dm}} \\
(\mathrm{K})\end{array}$ & $\begin{array}{l}\text { Desorp } \\
\mathrm{T}_{\mathrm{d}}(\mathrm{K})\end{array}$ & $\begin{array}{l}\mathrm{T}_{3 \mathrm{Dm}} \\
(\mathrm{K}) \\
{[\mathrm{a}]}\end{array}$ \\
\hline $\mathrm{CO} / \mathrm{MgO}$ & 0.3989 & 1 & 1.781 & 2.022 & $1.541[\mathrm{~b}]$ & 161 & 179 & 143 & 55 & 180 & 64.1 \\
\hline $\mathrm{CO} / \mathrm{NaCl}$ & 0.2848 & $1 / 2$ & 0.966 & 1.348 & $0.584[\mathrm{~b}]$ & 121 & 165 & 77 & 53 & 163 & 64.1 \\
\hline $\mathrm{CH}_{3} \mathrm{~F} / \mathrm{MgO}$ & 0.3989 & 1 & $2.21[\mathrm{c}]$ & $8.38[b]$ & $-3.96[b]$ & $345[\mathrm{~d}]$ & $292[\mathrm{e}]$ & $398[\mathrm{e}]$ & & & 131.3 \\
\hline $\mathrm{CH}_{3} \mathrm{~F} / \mathrm{NaCl}$ & 0.2848 & $1 / 2$ & $1.22[\mathrm{c}]$ & $7.39[\mathrm{~b}]$ & $-4.95[\mathrm{~b}]$ & $259[\mathrm{~g}]$ & $245[\mathrm{e}]$ & $274[\mathrm{e}]$ & & $103[\mathrm{f}]$ & 131.3 \\
\hline $\mathrm{CH}_{3} \mathrm{Cl} / \mathrm{MgO}$ & 0.3989 & $1 / 2$ & $3.59[\mathrm{c}]$ & 9.90 [b] & $-2.72[\mathrm{~b}]$ & $260[\mathrm{~g}]$ & $175[\mathrm{e}]$ & $346[\mathrm{e}]$ & & & 175.4 \\
\hline $\mathrm{CH}_{3} \mathrm{Cl} / \mathrm{NaCl}$ & 0.2848 & $1 / 2$ & $1.95[\mathrm{c}]$ & 8.26 [b] & $-4.36[\mathrm{~b}]$ & $195[\mathrm{~d}]$ & $170[\mathrm{e}]$ & $220[\mathrm{e}]$ & & & 175.4 \\
\hline $\mathrm{CH}_{3} \mathrm{Br} / \mathrm{MgO}$ & 0.3989 & $1 / 2$ & $4.73[\mathrm{c}]$ & $10.74[\mathrm{~b}]$ & $-1.28[\mathrm{~b}]$ & $200[\mathrm{~h}]$ & $87[\mathrm{e}]$ & $312[\mathrm{e}]$ & & & 199.8 \\
\hline $\mathrm{CH}_{3} \mathrm{Br} / \mathrm{NaCl}$ & 0.2848 & $1 / 2$ & $2.57[\mathrm{c}]$ & 8.58 [b] & $-3.44[\mathrm{~b}]$ & $140[\mathrm{~h}]$ & 107[e] & $173[\mathrm{e}]$ & & $120[\mathrm{i}]$ & 199.8 \\
\hline $\mathrm{CH}_{3} \mathrm{I} / \mathrm{MgO}$ & 0.3989 & $1 / 2$ & $6.81[\mathrm{c}]$ & 12.21 [b] & $1.41[\mathrm{~b}]$ & $130[\mathrm{j}]$ & 292[e] & 32 [e] & & & 206.7 \\
\hline $\mathrm{CH}_{3} \mathrm{I} / \mathrm{NaCl}$ & 0.2848 & $1 / 2$ & $3.69[\mathrm{c}]$ & 9.09 [b] & $-1.71[\mathrm{~b}]$ & $98[\mathrm{~d}$ & $146[\mathrm{e}]$ & $51[\mathrm{e}]$ & & & 206.7 \\
\hline
\end{tabular}

[a] See Ref. 5.

[b] Estimated; $\mathrm{p}_{\mathrm{u} / \mathrm{d}}=\mathrm{p}_{\text {ind }} \pm \mathrm{p}_{\text {nat }}$.

[c] Estimated; $p_{\text {ind }}=\alpha \cdot\left(\mathrm{p}_{\mathrm{CO}} / \alpha_{\mathrm{CO}}\right)$.

[d] Estimated; $\varepsilon_{\mathrm{b}}(\mathrm{A} / \mathrm{NaCl}$ or $\mathrm{MgO})=\varepsilon_{\mathrm{b}}(\mathrm{A} / \mathrm{MgO}$ or $\mathrm{NaCl}) \cdot\left[\varepsilon_{\mathrm{b}}(\mathrm{CO} / \mathrm{NaCl}\right.$ or $\mathrm{MgO}) / \varepsilon_{\mathrm{b}}(\mathrm{CO} / \mathrm{MgO}$ or $\left.\mathrm{NaCl})\right]$.

[e] Estimated; $\varepsilon_{\mathrm{b}} \pm\left[\mathrm{p}_{\mathrm{ind}}{ }^{2} / 2 \cdot \alpha\right]$.

[f] See Ref. 31

[g] See Ref. 32

[h] Estimated; linear interpolation from other $\varepsilon_{b}$ values.

[i] See Ref. 33

[j] See Ref. 34 


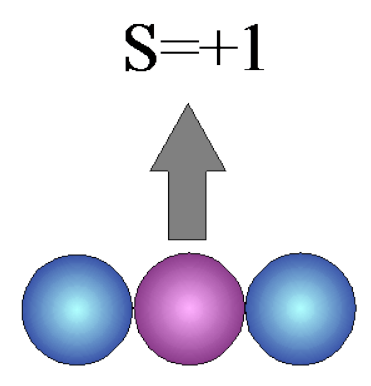

$\mu=\mu_{u}$

(i)

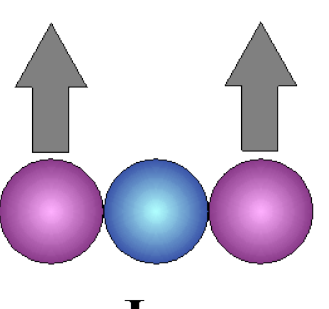

Juu

(i)

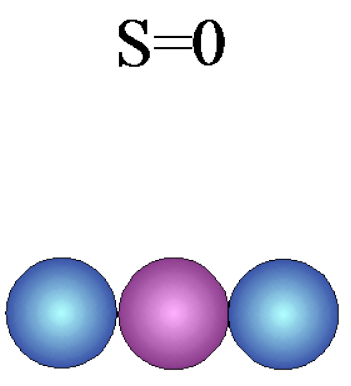

$\mu=0$

(ii)

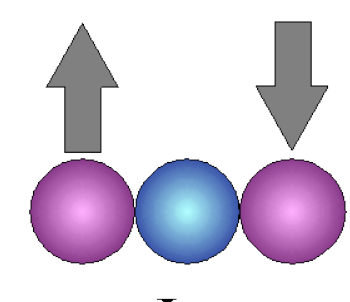

Jud

(ii)

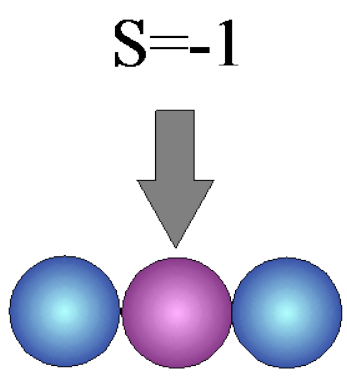

$\mu=\mu \mathrm{d}$

(iii)

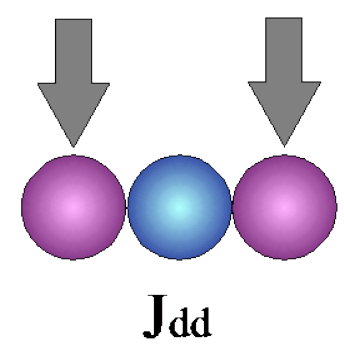

(iii) 


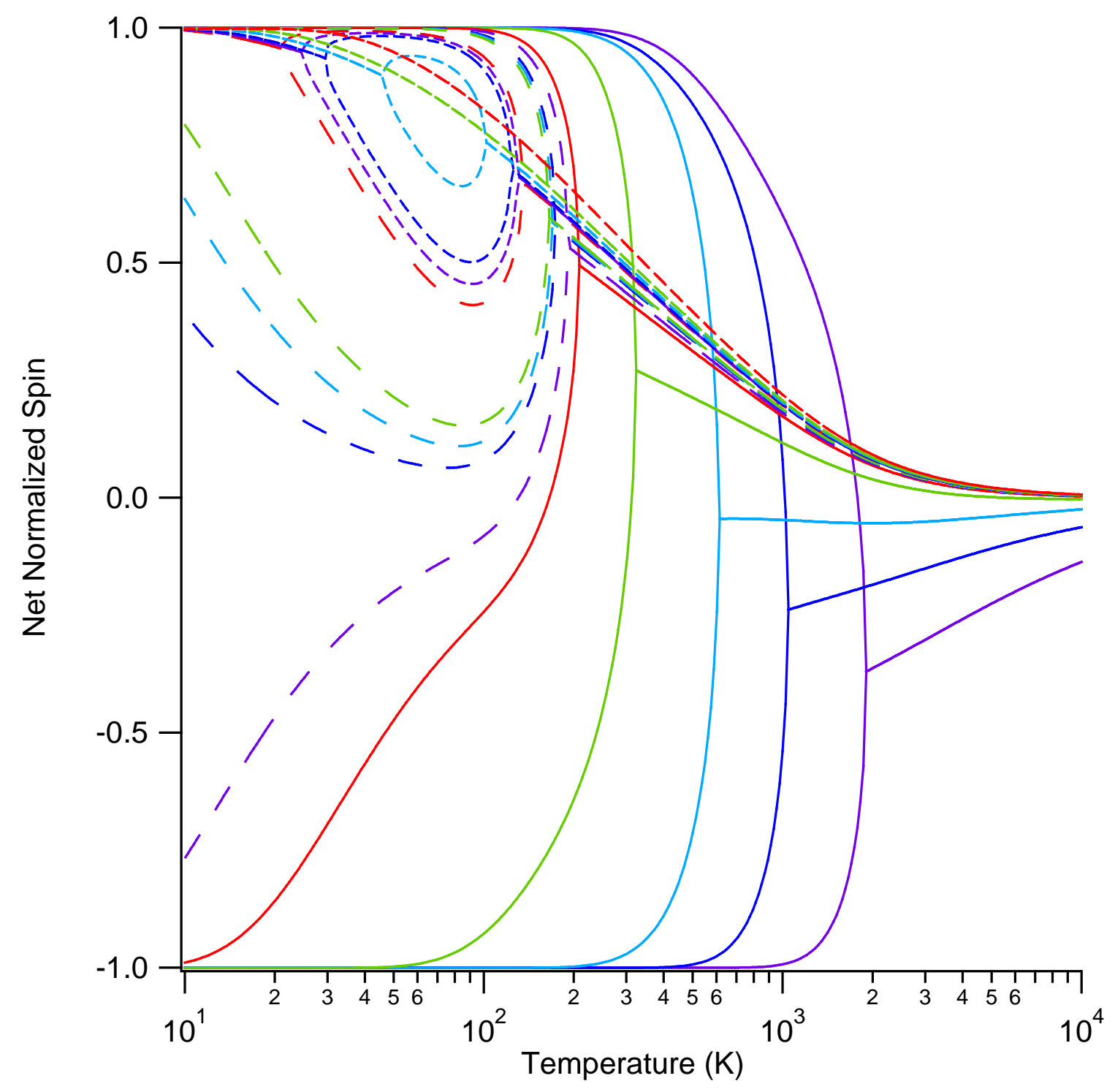

Figure 2 


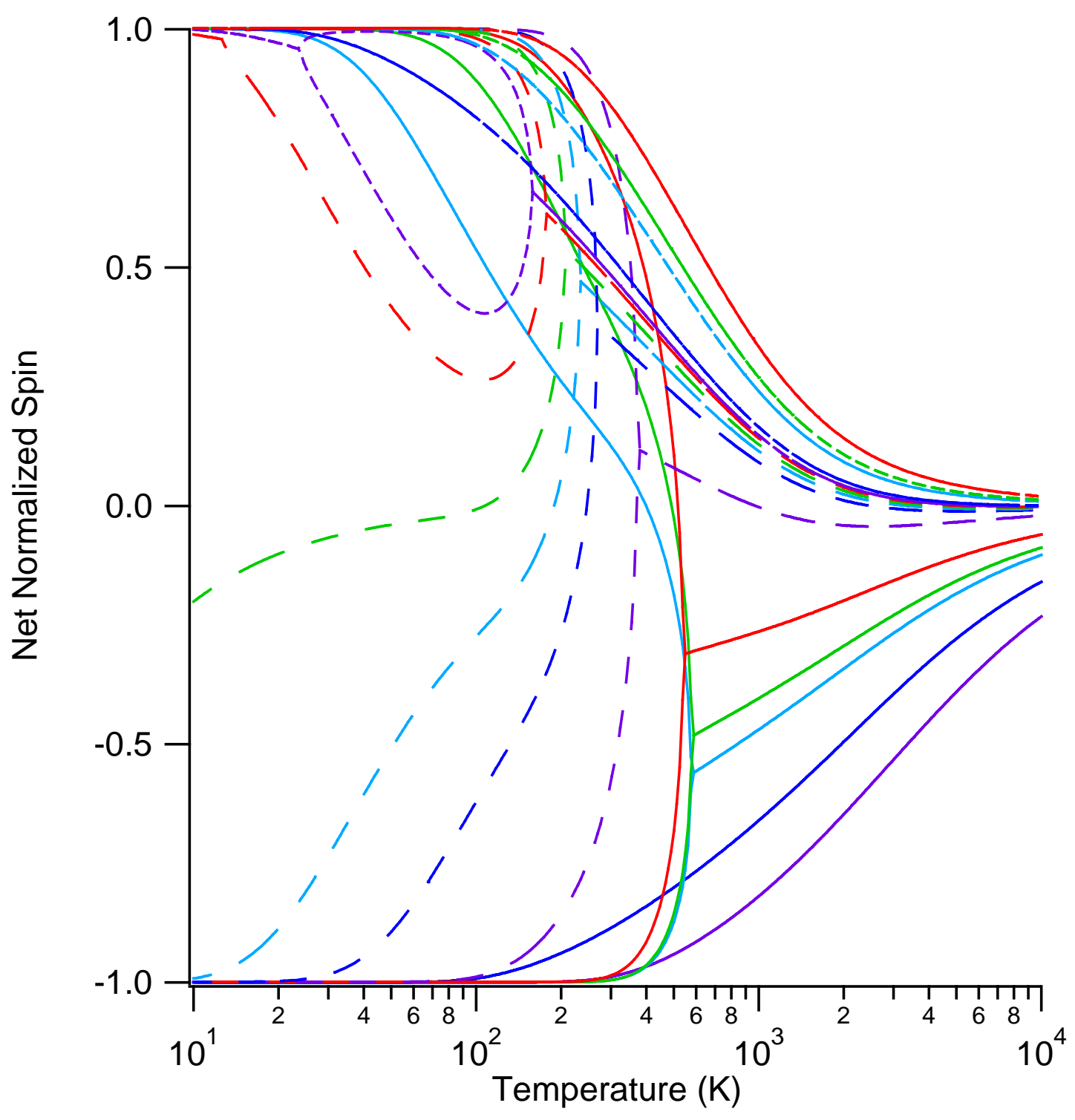

Figure 3

Burns, Surface Science 2003 

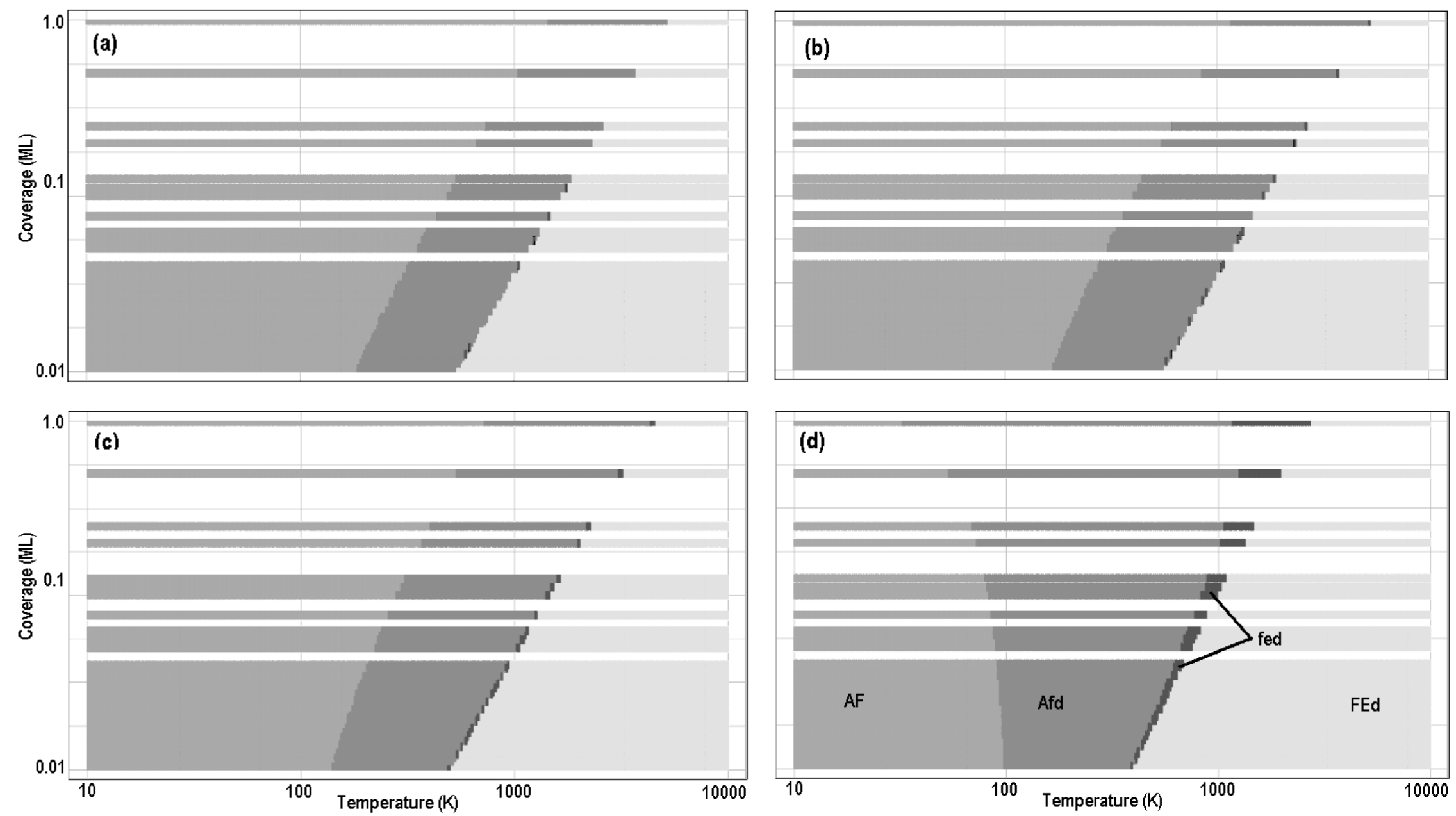

Figure 4

Burns, Surface Science 2003 

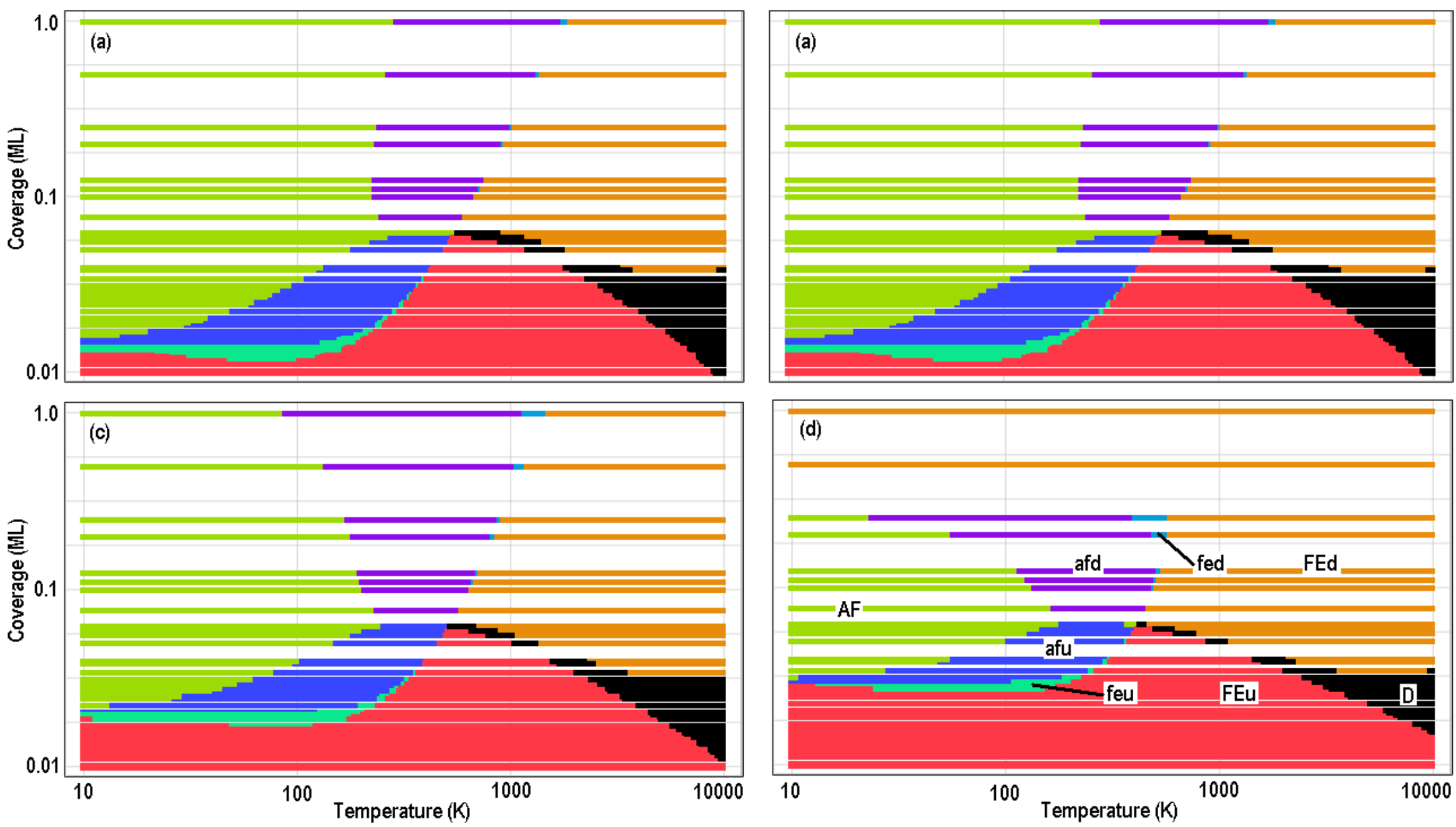

Figure 5

Burns, Surface Science 2003 